\title{
Modélisation de la Relation Pluie-Débit dans un Contexte de Gestion en Eau : Cas du Sous Bassin Versant du N'zi en Côte d'Ivoire
}

\author{
Fossou N'guessan Marie-Rosine, \\ Laboratoire Géosciences et Environnement (LEG) \\ UFR des Sciences et Gestion de L'Environnement \\ Université Nangui Abrogoua, Abidjan, Côte d'Ivoire
}

Soro Tanina Drissa, Laboratoire des Sciences et Technologies de l'Environnement (LSTE)

UFR Environnement Daloa, Côte d'Ivoire

Université Jean Lorougnon Guede

Soro Gbombélé, Orou Kotchi Rodrigue,

Laboratoire des sciences du sol, de l'eau et des géomatériaux UFR des Sciences de la Terre et des Ressources Minières Université Felix Houphouët Boigny Abidjan, Côte d'Ivoire

Goné Droh Lanciné, Laboratoire Géosciences et Environnement (LEG)

UFR des Sciences et Gestion de L'Environnement Université Nangui Abrogoua, Abidjan, Côte d'Ivoire

Doi:10.19044/esj.2020.v16n21p62～URL:http://dx.doi.org/10.19044/esj.2020.v16n21p62

\section{Résumé}

Cette étude s'inscrit dans un contexte de modélisation pluie-débit pour une optimisation des ressources en eau de surface. L'objectif principal est d'appliquer le modèle du Génie Rural (GR) sur le bassin versant de la région du N'zi afin de comprendre la relation pluie-débit dans ce bassin versant. L'utilisation des différents pas de temps mensuel (GR2M) et journalier (GR4J) ont permis de déterminer d'une part le meilleur modèle GR et d'autre part, les paramètres optimaux des stations de la zone d'étude. Une période de 18 ans a permis de déterminer les paramètres optimaux du bassin. Les critères de Nash obtenus pour le modèle GR4J varient de 40,80 à $67,40 \%$ pour les débits élevés, de 34,10 à $61 \%$ pour les débits moyens et de 8,80 à $30,90 \%$ pour les débits faibles. Cependant, les critères de Nash du modèle GR2M sont très faibles. Ils évoluent de 25,20 à 36,30\% pour les débits élevés, de 08,20 à 14,60 $\%$ pour les débits moyens et de -41 à $-31,40 \%$ pour les débits faibles. Le 
modèle GR4J simule mieux les écoulements du bassin que le modèle GR2M. En fait, les deux modèles simulent de façon acceptable la variation des débits observés en termes d'allure, mais ce sont les écarts qui existent entre les débits observés et simulés qui les distinguent. Le modèle GR4J serait mieux indiquer pour faire des prévisions sur les périodes d'inondation. Le coefficient d'échange X2 négatif, explique la raison pour laquelle le N'zi se vide dans le bassin de Bocanda, alors que la baisse de la pluviométrie est négligeable.

Mots-clés : Modélisation Pluie-Débit, Génie Rural (GR), Bassin Versant, Région Du N'zi, GR2M, GR4J 


\title{
Modeling of the Rain-Flow in a Water Management Context : Case of the N'zi Sub-Watershed in Côte d'Ivoire
}

\author{
Fossou N'guessan Marie-Rosine, \\ Laboratoire Géosciences et Environnement (LEG) \\ UFR des Sciences et Gestion de L'Environnement \\ Université Nangui Abrogoua, Abidjan, Côte d'Ivoire \\ Soro Tanina Drissa, \\ Laboratoire des Sciences et Technologies de l'Environnement (LSTE) \\ UFR Environnement Daloa, Côte d'Ivoire \\ Université Jean Lorougnon Guede \\ Soro Gbombélé, \\ Orou Kotchi Rodrigue, \\ Laboratoire des sciences du sol, de l'eau et des géomatériaux \\ UFR des Sciences de la Terre et des Ressources Minières \\ Université Felix Houphouët Boigny Abidjan, Côte d'Ivoire \\ Goné Droh Lanciné, \\ Laboratoire Géosciences et Environnement (LEG) \\ UFR des Sciences et Gestion de L'Environnement \\ Université Nangui Abrogoua, Abidjan, Côte d'Ivoire
}

\begin{abstract}
This study is part of a rain-flow modeling context for optimizing surface water resources. The main objective is to apply the flow simulation model, that of Rural Engineering (GR) on the watershed of the N'zi region in order to understand the rain-flow relationship in this watershed. The use of different monthly (GR2M) and daily (GR4J) time steps made it possible to identify, firstly the best GR model and scondly, the optimal parameters of the watershed. An 18 year period has therefore permitted to determine the optimal parameters of the watershed. The Nash criteria obtained for the GR4J model vary from 40.80 to $67.40 \%$ for high flow rates, from 34.10 to $61 \%$ for medium flow rates and from 8.80 to $30.90 \%$ for low flow rates. On the other hand, the Nash criteria of the GR2M model are very weak. They range from 25.20 to $36.30 \%$ for high bit rates, from 08.20 to $14.60 \%$ for medium bit rates and from -41 to $-31.40 \%$ for low bit rates. The GR4J model better simulates the flows of the watershed than the GR2M model. In fact, both models simulate in an
\end{abstract}


acceptable manner the variation of the observed flows in terms of pace, but it is the differences which exist between the observed and simulated flows which distinguish them. The GR4J model would be better suited for forecasting flood periods. The stall period should be relatively short for best results. The exchange coefficient X2 negative, explains why the N'zi empties into the Bocanda Basin, while the decrease in rainfall is negligible.

Keywords : Rain-Flow Modeling, Rural Engineering (GR), Watershed, N'zi Region, GR2M, GR4J

\section{Introduction}

Les travaux de Ouédraogo et al. (1998) sur les bassins versants en Afrique de l'Ouest et Centrale ont montré que la sécheresse semble avoir affecté la relation pluie-débit. Plusieurs auteurs tels que Savané et al. (2001), Goula et al. (2005), et Fossou et al. (2014) ont montré qu'en Côte d'Ivoire, le déficit pluviométrique observé sur plusieurs années consécutives s'est répercuté sur les écoulements des principaux fleuves et leurs affluents. Cette situation a provoqué une baisse considérable de leurs caractéristiques hydrologiques (débits moyens annuels, débits moyens journaliers maximums, débits d'étiage), conduisant ainsi, les populations à se retrouver face à un fort déficit hydrique pendant les saisons sèches. Ainsi, la connaissance de la variation du débit est aujourd'hui un moyen indispensable à la gestion des écosystèmes hydriques, notamment pour la prévision de débits, la gestion d'ouvrages de retenue et le dimensionnement d'ouvrages hydrauliques. En Afrique subsaharienne et tout particulièrement en Côte d'Ivoire, les données de débits existantes ont des séries souvent incomplètes ou encore non existantes surtout pour des cours d'eau non jaugés. Pour pallier ces insuffisances, le recours à la modélisation s'avère être nécessaire en vue de générer des séries de débits qui puissent permettre au gestionnaire de prendre des décisions pour la réalisation de certains projets de développement. En Côte d'Ivoire, plusieurs auteurs dont Ouédraogo (2001), Kouassi (2007) et Adja et al. (2019), ont eu recours à cet outil de modélisation de la relation pluie-débit. La compréhension des interactions pluie-débit s'avère indispensable vu son importance et son intérêt mondial. En particulier, cette compréhension a un intérêt privilégié dans le Centre-Est de la Côte d'Ivoire où la saison sèche est très rude.

L'objectif principal de cette étude est d'appliquer le modèle de simulation des débits, ceux du Génie Rural (GR) sur le bassin versant de la région du N'zi afin de comprendre la relation pluie-débit dans ce bassin versant. De façon spécifique, il s'agit d'utiliser les modèles GR2M et GR4J afin d'identifier d'une part le meilleur modèle GR, le temps de simulation et 
d'autre part d'identifier les paramètres optimaux des stations de la zone d'étude.

\section{Présentation de la zone d'étude}

La zone d'étude est située au Centre-Est de la Côte d'Ivoire entre les longitudes $4^{\circ} 40$ et $4^{\circ} 59$ Ouest et les latitudes $6^{\circ} 33$ et $7^{\circ} 27$ Nord avec une superficie de 5801,3 km² (Figure 1). Elle comprend 3 départements (Bocanda, Dimbokro et Kouassi-kouassikro), 5 sous-préfectures (N'zècrèzessou, Kouadio-Blèkro, Bengassou, Nofou et Djangokro) et plus de 200 villages.

Le réseau hydrographique couvre totalement la zone d'étude (Figure 1). Pendant la saison sèche la plupart des cours d'eau tarissent. Seuls les cours d'eau permanents subsistent. Le réseau intermittent est observé en période de crue (août, septembre, octobre). Le réseau permanent est constitué du N'zi et de ses principaux affluents dont le Kan et le Kasié. Le N'zi traverse la zone d'étude de l'Est jusqu'au Sud où il fait frontière avec le département de Bongouanou. Il poursuit son trajet en traversant la station de Dimbokro. Cette station est considérée comme l'exutoire dans cette étude. Le N'zi divise donc la zone d'étude en deux domaines hydrographiques. Il n'est pas navigable, car il forme un tracé en méandres et un régime permanent avec une période de crue et une période d'étiage. Le Kasié coule du Centre vers l'Ouest en direction du département de Dimbokro. Le Kan avec un tracé en méandres coule du Centre vers le Sud.

- Selon Yao et al. (1990 ; 1995), le climat de la région de type tropical humide est particulièrement chaud et relativement sec. La variation pluviométrique mensuelle moyenne de 1954 à 2010 dans la zone d'étude, présente 2 modes qui font ressortir les 4 saisons caractéristiques de cette région du pays (figure 2):

- la première saison des pluies est marquée par des pluies régulières et croissantes, d'avril à juin avec un maximum situé en juin ;

- une petite saison sèche marquée par une baisse des précipitations de juillet à août ;

- la seconde saison des pluies plus courte dure de septembre à octobre ;

- une grande saison sèche marquée par une baisse considérable des pluies. Elle s'étend de novembre à mars. La température moyenne sur une chronique de 50 ans (1951 à 2001) est de $26,93^{\circ} \mathrm{C}$. Les vents sont faibles en général, seul $l^{\prime}$ harmattan est important surtout dans la partie Nord de la région, mais son influence décroît rapidement vers le Sud.

- Sur le plan socio-économique, La zone d'étude est peuplée de 55138 habitants, selon le recensement général de la population et de l'habitat en 1998 publié par l'INS en 2001. Selon le bilan du conseil général, la population actuelle est estimée à plus de 100000 habitants. Les principales activités 
économiques de la région du N'zi sont l'agriculture, l'élevage et la pêche. Cependant, l'agriculture de cette zone connaît un déclin car aujourd'hui elle est appelée l'ancienne « boucle du Cacao ». La pêche est pratiquée de façon artisanale. Le développement de cette activité économique pourrait constituer une importante source de revenus pour les populations locales.

La région était jadis un grand centre commercial très actif, avec de grandes maisons de commerce. Cependant, les longues sécheresses et les crises successives sur les coûts du café et du cacao, ont occasionné la fermeture de ces maisons de commerce et le départ massif de leurs propriétaires ainsi que des populations vers les zones plus favorables du pays.

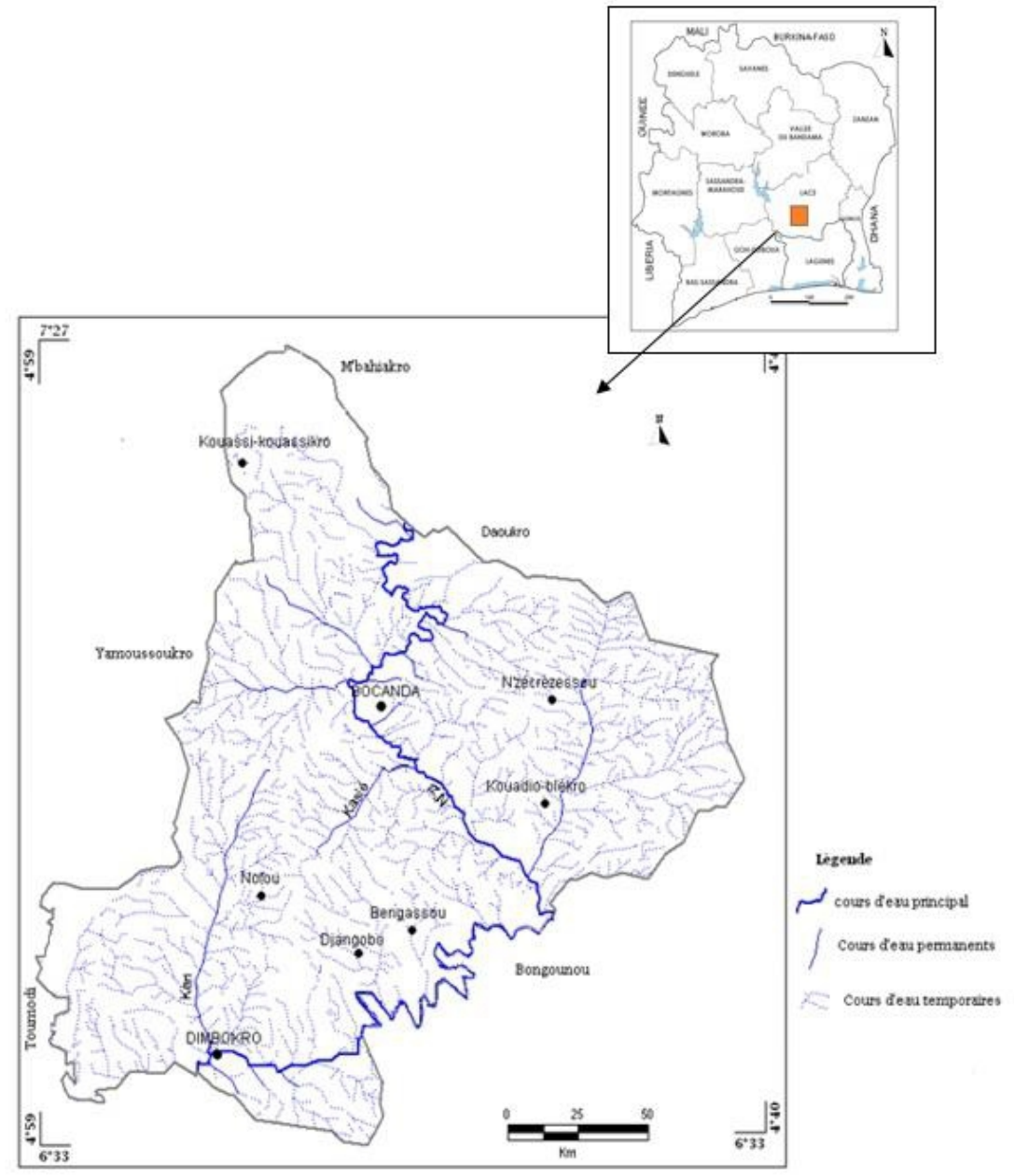

Figure 1 : Localisation de la zone d'étude 


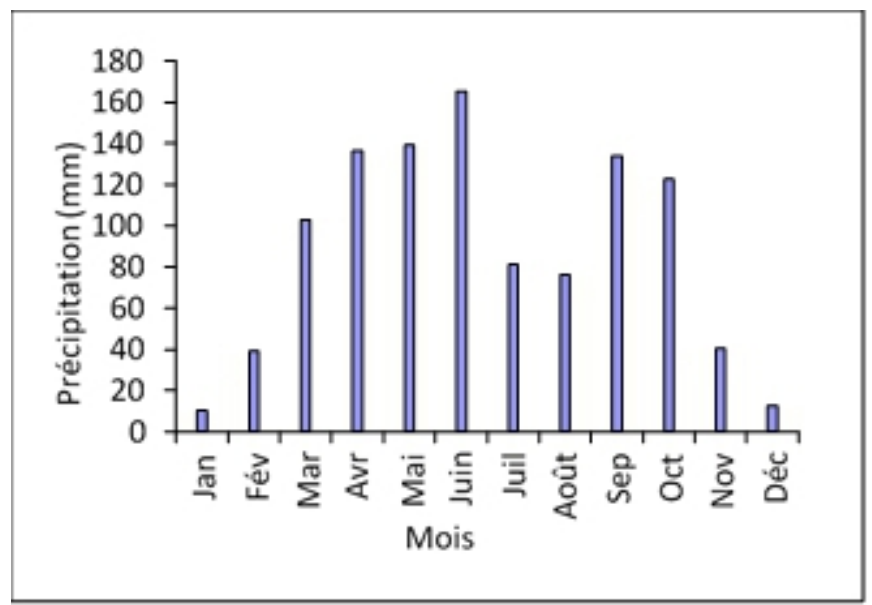

Figure 2 : Variation mensuelle de la pluviométrie de 1954 à 2010

L'évolution annuelle de la pluviométrie (figure 3) montre des courbes de tendance inclinées traduisant ainsi une légère baisse de la plu viométrie dans la zone d'étude.

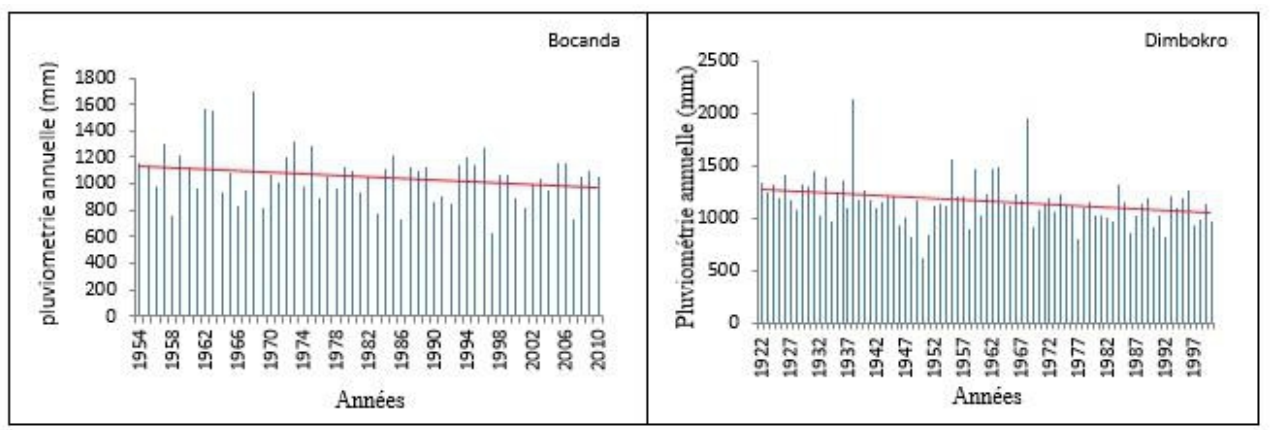

Figure 3 : Evolution de la pluviométrie annuelle totale à Bocanda, et Dimbokr

\section{Matériel et méthodes}

\subsection{Matériel}

La réalisation de cette étude a nécessité l'utilisation de nombreuses données d'origine diverses et un matériel adéquat.

\subsubsection{Données}

Les données utilisées sont de type hydroclimatique et hydrométrique. Toutes les données climatiques sont fournies par la Société d'Exploitation et de Développement Aéroportuaire, Aéronautique et Météorologique (SODEXAM). Le réseau des mesures pluviométriques comporte 2 stations météorologiques dont la station de Dimbokro, et de Bocanda. Les deux 2 chroniques de précipitations journalières n'ont pas la même taille. Elles vont de 1954 à 2010 à la station de Bocanda, de 1922 à 2000 à la station de Dimbokro. Une chronique de débits journaliers allant de 1955 à 1997 a été 
obtenue auprès de la sous-direction de l'hydrologie de la Direction de l'Hydraulique Humaine (DHH). Elle concerne les stations hydrométriques de Dimbokro, de Bocanda et de M'bahiakro.

Les données présentent beaucoup de lacunes. Cependant, les données hydrométriques comportent plus de lacunes par rapport aux données pluviométriques. Leur utilisation à l'état brut sans traitement est source d'erreur. De ce fait, elles doivent être comblées à l'aide de techniques appropriées.

\subsubsection{Correction des données pluviométriques}

Dans les chroniques pluviométriques analysées, les lacunes sont moins importantes et ne se situent pas sur des années successives. Elles se situent surtout entre décembre et avril à l'échelle mensuelle. Ces lacunes ont été comblées par la méthode des doubles masses (doubles cumuls). Le principe de cette méthode graphique consiste à vérifier l'homogénéité des valeurs mesurées de la station à tester en les corrélant avec celles de référence. Cette opération permet de combler les lacunes, de tester l'homogénéité et de critiquer les données brutes du réseau pluviométrique. Cette comparaison utilise, au pas de temps choisi, non pas les valeurs observées mais leur cumul (Meylan et Musy, 1998 in Riad 2003). L'évaluation des données manquantes à une station a été déterminée à partir des valeurs provenant de la station voisine présentant le meilleur coefficient de corrélation et ayant fonctionné durant le mois manquant. La formule la plus simple pour combler, sans erreur majeure les lacunes des séries, est de remplacer la valeur manquante par une moyenne pondérée par la tendance mensuelle des stations (Musy et Laglaine, 1992 in Riad 2003). Dans cette étude, la station de référence choisie est celle de Dimbokro car elle présente la plus longue chronique avec moins de lacunes.

\subsubsection{Correction des données hydrométriques}

Les données de débits des trois stations comportent beaucoup de lacunes. Cependant, les données de la station de Dimbokro présentent moins de lacunes que les deux autres stations. Dans la littérature, plusieurs techniques existent pour reconstituer les données manquantes. En effet, dans le cas de stations hydrométriques placées sur un même cours d'eau (transposition géographique, conservation des volumes, etc.), les critères de proportionnalité analogues peuvent être utilisés. Les méthodes basées sur l'analyse des régressions linéaires et des corrélations (relation pluie-pluie ou pluie-débit, relations inter-postes) peuvent également être utilisées à cet effet (Musy et Higy, 2003 in Koffi 2007). Dans cette étude, nous avons utilisé la méthode du critère de proportionnalité analogue pour reconstituer les données manquantes de débits des stations. Les différentes surfaces des sous-bassins ont été calculées et les rapports ont été utilisés (équations 1 à 5) ci-dessous. En 
régime tropical, les débits d'étiage sont très faibles voire nuls et peuvent être remplacés, très souvent, par les moyennes interannuelles sans avoir recours à des corrélations mensuelles ou aux débits journaliers (Ardoin-Bardin, 2004). Cette méthode a été utilisée en complément de la première. La méthode du critère de proportionnalité analogue donne la relation linéaire du type (équation 1)

$$
\frac{\mathrm{Q}_{1}}{\mathrm{~S}_{1}}=\frac{\mathrm{Q}_{2}}{\mathrm{~S}_{2}}
$$

où, Q1 et Q2 correspondent respectivement aux débits relevés à l'exutoire de la station 1 et 2 ; et $\mathrm{S} 1$ et $\mathrm{S} 2$ les superficies des bassins versants. Ainsi, les relations suivantes (équations 2 à 4 ) ont été obtenues entre les stations de N'Zianoua, Bocanda, M'Bahiakro et Dimbokro connaissant les superficies de leurs bassins versants. En effet, les données de la station de N'Zianoua ont été utilisées pour combler les lacunes des trois autres stations parce qu'elles présentent moins de lacunes que les trois autres. En rappel, cette station est incluse dans le bassin du N'zi, elle est située en aval des trois autres.

$$
\begin{array}{ll}
\mathrm{Q}_{\mathrm{M} / \text { Bahiakro }}=0,44 \mathrm{Q}_{\mathrm{N} / \text { Zianoua }} & \text { Eq. } 2 \\
\mathrm{Q}_{\text {Bocanda }}=0,58 \mathrm{Q}_{\mathrm{N} / \text { ianoua }} & \text { Eq. } 3 \\
\mathrm{Q}_{\text {Dimbokro }}=0,68 \mathrm{Q}_{\mathrm{N} / \text { Zianoua }} & \text { Eq. } 4
\end{array}
$$

\subsection{Méthode}

\subsubsection{Le modèle Génie Rural (GR)}

Le modèle GR est un modèle à l'échelle d'un bassin versant et ne tient pas compte des états de surface. Contrairement à certains modèles globaux (Modèle de gestion des Eaux pluviales: SWMM) qui sont à l'échelle de plusieurs bassins et qui prennent en compte les états de surface.

Dès le début des années 1980, l'Institut National de Recherche en Sciences et Technologies pour l'Environnement et l'Agriculture, (Irstea, Ex. Cemagref) a commencé à développer des modèles hydrologiques du Génie Rural (GR) permettant de faire le lien entre la lame d'eau précipitée sur un bassin versant et son débit à l'exutoire (Michel, 1983). Les modèles GR ont été développés par pas de temps de fonctionnement. Le modèle est donc spécifique pour les pas de temps : annuel (GR1A), mensuel (GR2M) et journalier (GR4J). Les pas de temps mensuel et journalier ont été choisis pour cette étude parce qu'ils permettent de déterminer les paramètres optimaux des bassins étudiés. La période minimale pour l'utilisation de ce modèle est de 12 ans. Il est disponible sur le site du CEMAGREF.

\subsubsection{Application du modèle GR2M}

Le modèle GR2M est constitué (Mouelhi, 2003) :

- d'un réservoir de production qui régit la fonction de production et est caractérisé par sa capacité maximale ;

- d'un réservoir « eau gravitaire » régissant la fonction de transfert. 
Le mode de fonctionnement du modèle GR2M est décrit par la figure 4.

Le modèle peut être expliqué de la façon suivante :

- une fraction de la pluie mensuelle qui participe à l'écoulement direct;

- le niveau d'un réservoir qui représente le stock d'eau et évolue en fonction de la fraction de pluie restante et de l'évapotranspiration potentielle par l'intermédiaire d'une évapotranspiration réelle ;

- une vidange progressive de ce stock d'eau qui produit l'écoulement retardé.

Ce modèle de bilan d'eau contient 2 paramètres (X1 et X2). Le premier paramètre (X1) représente la capacité maximale du réservoir « sol ». Le deuxième paramètre $(\mathrm{X} 2)$ représente le paramètre d'échange souterrain au niveau du réservoir « eau gravitaire ». En effet, un nombre de deux paramètres libres dans un modèle conceptuel global est largement suffisant à l'échelle de temps mensuel (Mouelhi, 2003).

- Le fondement mathématique du modèle GR2M se présente comme suit : on pose que :

$$
\varphi=\tan h\left(\frac{P}{X 1}\right)
$$

Eq. 5

- Le niveau $S$ (stock d'eau) dans le réservoir devient $S 1$ sous l'action de la pluie $P$ (opération 1 de la figure 4) et est donné par l'équation 6 :

$$
S 1=\frac{\mathrm{S}+\mathrm{X} 1 \varphi}{1+\varphi \frac{S}{X 1}} \quad \text { Eq. } 6
$$

où le paramètre $X 1$, capacité du réservoir, est positif et exprimé en $\mathrm{mm}$. Il s'ensuit une contribution à l'écoulement $P 1$ (opération 2 de la figure 4) par l'équation 7.

$$
P 1=P+S-S 1
$$

En posant $\psi=\tanh \left(\frac{E}{x 1}\right)$, le niveau $S 1$ devient $S 2$ sous l'effet de l'évapotranspiration $\mathrm{E}$ (opération 3 de la figure 4) et est exprimé par l'équation 8 :

$$
S 2=\frac{S 1(1-\psi)}{1+\psi\left(1-\frac{S 1}{X 1}\right)}
$$




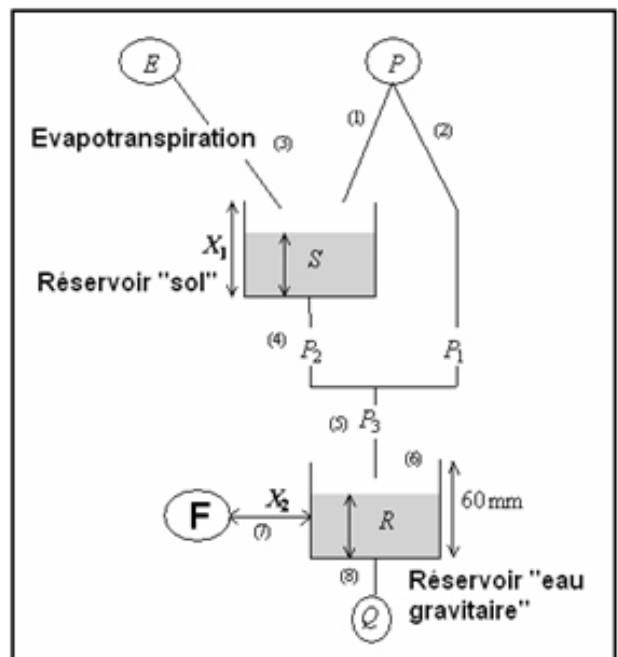

Figure 4 : Schéma de fonctionnement du modèle GR2M (Mouelhi, 2003)

- Le réservoir «sol » se vidange ensuite en une percolation $P_{2}$ (opération 4 de la figure 4) et son niveau $\mathrm{S}$, prêt pour les calculs du mois suivant, est alors donné par l'équation 9 :

$$
\begin{gathered}
S=\frac{S 2}{\left[1+\left(\frac{S 2}{X 1}\right)\right]^{1 / 3}} \\
P_{2}=S_{2}-S
\end{gathered}
$$

- La pluie totale $P_{3}$ qui atteint le réservoir de routage (opération 5 de la figure 4) est donnée par l'équation 10 :

$$
P_{3}=P_{1}+P_{2}
$$

Eq. 10

- Le réservoir $\mathrm{R}$, dont le niveau en début du mois est $R$ devient $R_{1}$ (opération 6 de la figure

5) est donné par l'équation 11 :

$$
R_{1}=R+P_{3}
$$

- L'échange, $\mathrm{F}$ (opération 7 de la figure 4), qui agit sur le réservoir $\mathrm{R}$ est donnée par l'équation 12 :

$$
F=\left(X_{2}-1\right) R_{1}
$$

Eq. 12

Le paramètre $X_{2}$ est positif et adimensionnel.

- Le niveau dans le réservoir devient $\mathrm{R}_{2}$ et son expression est donnée par l'équation 13 :

$$
R_{2}=X_{2} R 1
$$

- Le réservoir, de capacité fixée (60 $\mathrm{mm}$ dans le cas de la figure 4 ), se vidange suivant une fonction quadratique (opération 8 de la figure 4) et le débit est exprimé par l'équation 14 :

$$
Q=\frac{R_{2}^{2}}{R_{2}+60}
$$


L'application du modèle consiste à caler les deux paramètres X1 et X2 sur une période test.

Ensuite à valider et à vérifier les résultats sur deux périodes différentes.

Le principe est donc basé sur des calages successifs sur l'ensemble de la période où les données de pluie et de débit sont disponibles. Le modèle a été conçu à partir de l'outil Excel, ce qui permet de faire les calculs.

L'application du modèle consiste à caler les deux paramètres X1 et X2 sur une période test.

Ensuite à valider et à vérifier les résultats sur deux périodes différentes.

Le principe est donc basé sur des calages successifs sur l'ensemble de la période où les données de pluie et de débit sont disponibles. Le modèle a été conçu à partir de l'outil Excel, ce qui permet de faire les calculs.

\subsubsection{Application du modèle GR4J}

Le modèle GR4J (modèle du Génie Rural à 4 paramètres Journalier) est un modèle pluie-débit global à quatre paramètres (Makhlouf, 1994). Sa structure associe un réservoir de production et un réservoir de routage, des hydrogrammes unitaires ainsi qu'une fonction d'ouverture sur l'extérieur non atmosphérique permettant de simuler l'ensemble du comportement hydrologique du bassin (figure 5). Le modèle GR4J a certaines fonctions communes avec le modèle GR2M (par exemple la fonction de production liée au réservoir sol). Son module de routage est cependant plus sophistiqué qu'au pas de temps mensuel. Ses quatre paramètres sont les suivants :

- $X 1$ : capacité maximale du réservoir de production $(\mathrm{mm})$;

- $X 2$ : coefficient d'échanges souterrains (mm);

- $X 3$ : capacité à un jour du réservoir de routage $(\mathrm{mm})$;

- $X 4$ : temps de base de l'hydrogramme unitaire $H U 1$ (j).

- Neutralisation

La première opération est la neutralisation de $P$ par $E$ pour déterminer une pluie nette $P n$ et une évapotranspiration nette $E n$ calculée par les équations 15 et 16 :

Si $P \geq E$ alors $P n=P-E$ et $E n=0$

Eq. 15

Si $P<E$, alors $P n=0$ et $E n=E-P k$

Eq. 16

- Fonction de rendement

Dans le cas où $P n$ est différente de zéro, une partie $P s$ de $P n$ alimente le réservoir de

Production et est calculée par l'équation 17 :

$$
P S=\frac{X_{1}\left(1-\left(\frac{S}{X_{1}}\right)^{2}\right) \cdot \tanh \left(\frac{P n}{X_{1}}\right)}{1+\frac{S}{X_{1}} \cdot \tanh \left(\frac{P n}{X_{1}}\right)}
$$

où $X 1(\mathrm{~mm})$ est la capacité maximale du réservoir de production et $S$ le contenu du réservoir de production au début du jour. Dans le cas contraire, lorsque En 
est différente de zéro, une quantité d'évaporation $E s$ est retirée du réservoir de production. Es est donnée par l'équation 18 :

$$
E_{S}=\frac{S \cdot\left(2-\frac{S}{X_{1}}\right) \cdot \tanh \left(\frac{E_{n}}{X_{1}}\right)}{1+\left(1-\frac{S}{X_{1}}\right) \cdot \tanh \left(\frac{E_{n}}{X_{1}}\right)}
$$

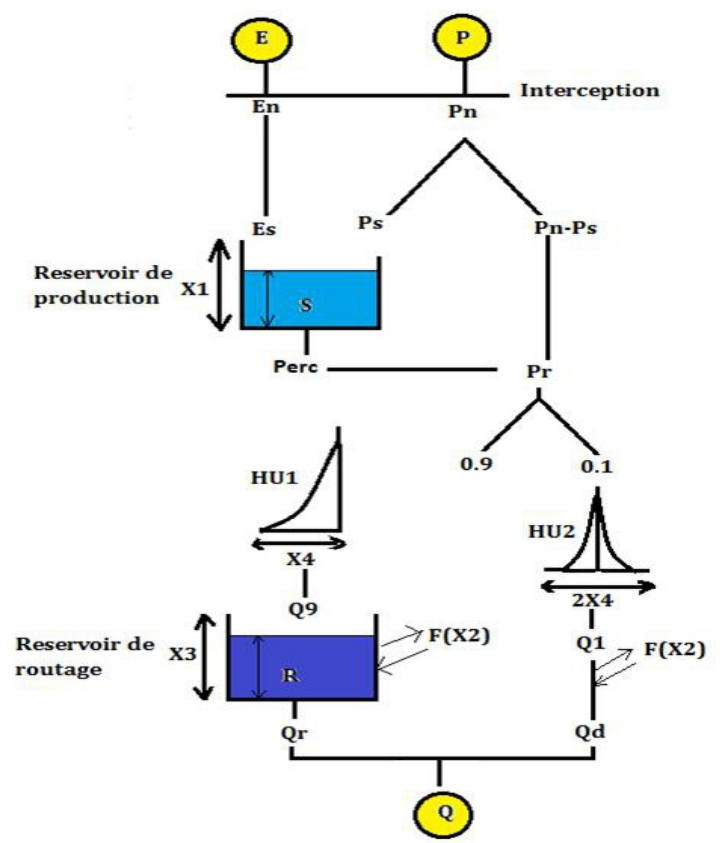

Figure 5 : Schéma de la structure du modèle GR4J (Traoré et al., 2014)

Le contenu du réservoir qui résulte de ces opérations est donné par l'équation 19 :

- Percolation

$$
S^{\prime}=S+P S-E s
$$

Eq. 19

Une percolation Perc issue du réservoir de production est alors calculée par l'équation 20 :

$$
\operatorname{Perc}=S\left\{1-\left[1+\left(\frac{4 S}{9 \times 1}\right)^{4}\right]^{\frac{-1}{4}}\right\}
$$

Par suite, le contenu du réservoir devient :

$$
S_{1}=S^{\prime}-\text { Perc }
$$

et la quantité d'eau $\operatorname{Pr}$ qui atteint finalement la partie routage du modèle est donnée par 1'équation 22 :

$$
\operatorname{Pr}=\operatorname{Perc}+(P n-P s)
$$

- Hydrogrammes unitaires

$\operatorname{Pr}$ est divisée en deux composantes d'écoulement, 90\% étant routés par un hydrogramme unitaire $H U 1$ et un réservoir de routage et $10 \%$ par un 
hydrogramme unitaire symétrique $H U 2$. $H U 1$ et $H U 2$ dépendent du même paramètre $X 4$, temps de base de $H U 1$ exprimé en jours. Les ordonnées des hydrogrammes sont calculées à partir des courbes en $\mathrm{S}$ notées respectivement SH1 et $S H 2$, qui correspondent aux fonctions cumulées de l'hydrogramme. $S H 1$ et $S H 2$ sont définies en fonction du temps par les équations 23 et 24 :

$$
\begin{gathered}
S H 1(t)=\left\{\begin{array}{ccc}
0 & \text { si } t \leq 0 \\
\left(\frac{t}{X 4}\right)^{\frac{5}{2}} & \text { si } & 0<t<X 4 \\
1 & \text { si } & \text { si } t \geq X 4
\end{array}\right. \\
S H 2(\mathrm{t})=\left\{\begin{array}{ccc}
0 & \text { si } 0<t<X 4 \\
\frac{1}{2}\left(\frac{\mathrm{t}}{\mathrm{X} 4}\right)^{\frac{5}{2}} & \text { si } \mathrm{X} 4<t<2 X 4 \\
\frac{1}{2}\left(2-\frac{\mathrm{t}}{\mathrm{X} 4}\right)^{\frac{5}{2}} & \text { si } & \mathrm{t} \geq 2 \mathrm{X} 4 \\
1 & \text { si } &
\end{array}\right.
\end{gathered}
$$

Les ordonnées de $H U 1$ et $H U 2$ sont alors calculées par les équations 25 et 26: $U H 1(\mathrm{j}) \square \square S H 1(\mathrm{j}) \square \square S H 1(\mathrm{j} \square 1)$ Eq. 25 $U H 2(\mathrm{j}) \square \square S H 2(\mathrm{j}) \square \square S H 2(\mathrm{j} \square 1)$ Eq. 26 où $j$ est un entier. A chaque pas de temps $k$, les sorties $Q 9$ et $Q 1$ des deux hydrogrammes correspondent à la convolution des pluies antérieures par la clé de répartition donnée par l'hydrogramme discrétisé et sont calculées par les équations 27 et 28 :

$Q 9(i)=0,9 \cdot \sum_{k=l}^{L} H U 1(k) \cdot \operatorname{Pr}(i-k+1)$

$Q 1(i)=0,1 \cdot \sum_{k=l}^{m} H U 2(k) \cdot \operatorname{Pr}(i-k+1)$

Eq. 28

où $1=\operatorname{int}(\mathrm{X} 4)+1$ et $\mathrm{m}=\operatorname{int}(2 . \mathrm{X} 4)+1$, avec int (.) désignant la partie entière.

- Fonction d'échange avec l'extérieur non atmosphérique

Un échange souterrain en eau est calculé par l'équation 29 :

$F=X_{2}\left(\frac{R}{X 3}\right)^{\frac{7}{2}}$

où $R$ est le niveau dans le réservoir en début de pas de temps, $X 3$ la capacité à un jour du réservoir et $X 2$ le coefficient d'échange en eau qui peut être positif dans le cas d'apports, négatif dans le cas de pertes vers des nappes profondes ou nul. En fait, l'interprétation physique de cette fonction d'échange n'est pas directe.

- Réservoir de routage

Le niveau dans le réservoir de routage est modifié en ajoutant la sortie $Q 9$ de l'hydrogramme $H U 1$ et $F$ définit par l'équation 30 :

$R^{\prime}=\max (0 ; R+Q 9+F)$

Eq. 30

Il se vidange ensuite en une sortie $Q r$ donnée par l'équation 31 :

$$
Q r=R \cdot\left\{1-\left[1+\left(\frac{R}{X 3}\right)^{4}\right]^{\frac{-1}{4}}\right\}
$$

Et le niveau dans le réservoir devient :

$$
R l=R^{\prime}-Q r
$$

- Ecoulement total 
La sortie $Q 1$ de l'hydrogramme $H U 2$ est soumise au même échange pour donner la composante d'écoulement $Q d$ définit par l'équation 33 :

$Q d=\max (0 ; Q 1+F)$

Eq. 33

Le débit total $Q$ est alors donné par l'équation 34 :

$Q k=Q r+Q d$

Eq. 34

L'application du modèle GR4J est la même que celle du modèle GR2M.

\subsubsection{Identification du meilleur modèle GR et de la période de simulation}

L'identification du meilleur modèle consiste à faire une étude test. Il s'agit d'utiliser les modèles GR4J et GR2M sur une même période en faisant varier la durée de calage. Cette application permet dans un premier temps de connaître lequel des deux modèles donne les meilleurs résultats. Dans un second temps, d'identifier l'influence de la période de calage sur la détermination des meilleurs paramètres optimaux.

Cette étude test a été faite sur le sous bassin de Bocanda parce qu'il présente une situation préoccupante (Fossou et al. 2014). Par ailleurs, l'écoulement du N'zi à Dimbokro présente les mêmes caractéristiques que celui du N'zi à Bocanda.

\subsubsection{Détermination des paramètres optimaux des bassins étudiés}

La détermination des paramètres optimaux des bassins a été faite sur une période de simulation comprise entre 1975 et 1993 soit 18 ans. Cette période a été choisie parce que sa série pluviométrique comporte moins de lacunes. Par ailleurs, la simulation avec des données plus récentes favorise des prévisions plus fiables.

Les phases de validation et de vérification ont été faites sur la version exécutable du modèle. Les paramètres optimaux ont été imposés au modèle pour vérifier les valeurs de validité obtenues lors du calage. Cette étape permet en outre, de détecter des erreurs dans les données utilisées.

\subsubsection{Critère de validité des résultats du modèle}

La fonction de critère ou critère numérique est l'objet numérique de référence lors du calage (automatique ou manuel) d'un modèle (Mouelhi, 2003). Cette fonction doit tendre vers une valeur donnée (minimum, maximum ou constante) lorsque les valeurs calculées tendent vers les valeurs observées. Il existe différentes formes de fonctions critères (critère de Nash, critère de Fortin, critère du bilan volumique, etc.). Le critère d'optimisation dans le cadre de cette étude est porté sur le critère de Nash. En effet, plusieurs études comparatives entre différentes formes de critères ont été effectuées et ont montré que le critère de Nash s'impose comme celui qui, globalement, permet d'accéder au meilleur calage (Servat et al., 1989 in Mouelhi, 2003 ; Perrin, 2000). Ainsi, il vérifie l'aptitude du modèle à reproduire respectivement les débits maximums, les débits d'étiage et les débits moyens. 
Le critère de Nash est défini comme suit (équation 35-37) :

$\operatorname{Nash} Q=100\left[1-\frac{\sum\left(\mathrm{Q}_{i}^{\text {obs }}-Q_{i}^{\text {cal }}\right)^{2}}{\sum\left(\overline{Q_{1}^{\text {obs }}}-Q_{i}^{\text {cal }}\right)^{2}}\right]$

Eq. 35

$\operatorname{Nash} \sqrt{ } Q=100\left[1-\frac{\sum\left(\sqrt{Q_{i}^{\text {obs }}}-\sqrt{Q_{i}^{\text {cal }}}\right)^{2}}{\sum\left(\sqrt{\mathrm{Q}_{1}^{\text {obs }}}-\sqrt{Q_{i}^{\text {cal }}}\right)^{2}}\right]$

Eq. 36

Nashlog $Q=100\left[1-\frac{\sum\left(\log Q_{i}^{\text {obs }}-\log Q_{i}^{\text {cal }}\right)^{2}}{\sum\left(\overline{\log Q_{1}^{\text {obs }}}-\log Q_{i}^{\text {cal }}\right)^{2}}\right]$

Eq. 37

avec :

$Q_{i}^{o b s}$ : débit journalier ou mensuel observé au jour i ou au mois i;

$\underline{Q_{i}^{c a l}}$ : débit journalier ou mensuel calculé au jour i ou au mois i;

$\overline{Q_{l}^{o b s}}$ : débit moyen observé au cours de la période de simulation.

La performance du modèle peut être jugée selon les valeurs prises par le critère de Nash

(Kachroo, 1986 in Koffi et al., 2007):

- Nash $\square \square 90 \%$ : le modèle est excellent ;

- 80\% < Nash < $90 \%$ : le modèle est très satisfaisant ;

- 60\% < Nash < 80\% : le modèle est satisfaisant ;

- Nash $<60 \%$ : le modèle est mauvais.

L'analyse des résultats de simulation est portée sur les performances des modèles au calage et à la validation. En effet, les performances au calage sont moins révélatrices des réelles capacités de simulation des modèles. Celles-ci sont mieux exprimées par la validation (Perrin, 2000). Le tableau I donne un exemple des valeurs des paramètres obtenus par les deux modèles sur un large échantillonnage par Perrin et al. (2007).

Tableau I : Valeurs des paramètres du modèle GR2M et GR4J obtenues sur un large échantillon de bassins versants (Perrin et al., 2007)

\begin{tabular}{ccc}
\hline Paramètres $(\mathbf{m m})$ & $\begin{array}{c}\text { Intervalle de confiance } \\
\mathbf{8 0 \%} \text { GR2M }\end{array}$ & $\begin{array}{c}\text { Intervalle de confiance } \\
\mathbf{8 0 \%} \text { GR4J }\end{array}$ \\
\hline X1 & 140 à 2640 & 100 à1200 \\
X2 (-) & 0,21 à 1,31 & -3 à 5 \\
X3 & & 20 à 300 \\
X4 & & 1,1 à 2,9 \\
\hline
\end{tabular}

\section{Résultats et discussion}

\subsection{Résultats}

Les résultats présentés concernent l'identification du meilleur modèle GR et à la détermination des paramètres optimaux des bassins de Bocanda et de Dimbokro. 


\subsubsection{Identification du meilleur modèle et de la période de simulation}

L'étude test a d'abord été faite avec le GR4J (plus complet) puis avec le GR2M. Les résultats consistent à l'interprétation des tableaux et graphes.

\subsubsection{Modèle GR4J}

Le tableau II montre les résultats de calage et de validation du modèle GR4J à la station de Bocanda. Le tableau présente la variation des paramètres optimaux et des critères de validité. L'analyse du tableau montre que la variation de la période de calage n'a pas d'influence significative sur la variation des paramètres $\mathrm{X} 2, \mathrm{X} 3$ et $\mathrm{X} 4$. Cependant, le paramètre $\mathrm{X} 1$ est instable (figure 6 A). Les critères de Nash ont la même allure (figure 6 B), cela signifie qu'ils évoluent dans le même sens. Ils diminuent au fur et à mesure que la période de calage augmente.

Tableau I : Calage et validation du modèle GR4J sur le sous bassin de Bocanda

\begin{tabular}{cc|cccc|ccc}
\hline \multirow{2}{*}{$\begin{array}{c}\text { Phase de } \\
\text { calage }\end{array}$} & $\begin{array}{c}\text { Phase de } \\
\text { Validation }\end{array}$ & $\mathbf{X}$ & $\mathbf{X}$ Paramètres & $\mathbf{X 2}$ & $\mathbf{X 3}$ & $\mathbf{X 4}$ & \multicolumn{3}{c}{$\begin{array}{c}\text { Critères de validation }(\%) \\
\text { Nash }\end{array}$} & $\begin{array}{c}\text { Nash } \\
\text { (hQ) }\end{array}$ & $\begin{array}{c}\text { Nash } \\
\text { (lnQ) }\end{array}$ \\
\hline $1961-1963$ & $1964-1990$ & 1581,07 & $-2,91$ & 101,61 & 4,320 & $\mathbf{6 7 , 4 0}$ & $\mathbf{6 1 , 0 0}$ & $\mathbf{3 0 , 9 0}$ \\
$1961-1964$ & $1965-1990$ & 1649,22 & $-3,10$ & 110,73 & 2,40 & 52,70 & 47,20 & 19,60 \\
$1961-1965$ & $1966-1990$ & 1532,20 & $-3,23$ & 97,81 & 5,26 & 49,70 & 44,40 & 16,10 \\
$1961-1966$ & $1967-1990$ & 1511,14 & $-3,77$ & 104,29 & 5,27 & 40,90 & 35,30 & 9,90 \\
$1961-1967$ & $1968-1990$ & 1367,12 & $-3,84$ & 88,47 & 3,28 & 40,80 & 34,10 & 8,80 \\
$1961-1968$ & $1969-1990$ & 1507,84 & $-3,21$ & 82,07 & 4,19 & 47,60 & 44,40 & 20,70 \\
$1961-1969$ & $1970-1990$ & 1435,85 & $-3,63$ & 86,46 & 4,20 & 46,80 & 42,70 & 19,20 \\
$1961-1970$ & $1971-1990$ & 1192,18 & $-5,30$ & 100,21 & 5,06 & 45,00 & 40,10 & 14,30 \\
\hline
\end{tabular}

Mais 1968 est l'année où la pluviométrie est maximale sur la période d'observation. Au-delà de cette année, les critères de Nash recommencent à décroitre. Ainsi, une période de simulation courte donnera de meilleurs résultats. 
Figure 6 : Evolution des paramètres (A) et des critères de validité (B) du modèle GR4J à la station de Bocanda

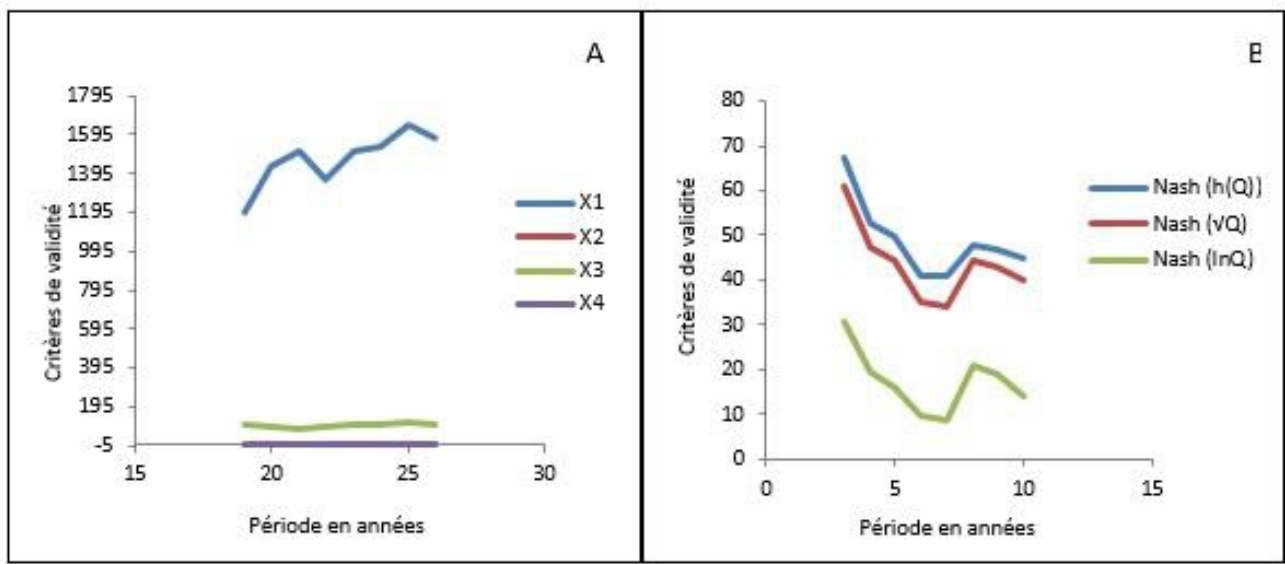

Les critères de Nash évoluent de 40,80 à $67,40 \%$ pour Nash(hQ), de 34,10 à $61 \%$ pour Nash $(\sqrt{ } \mathrm{Q})$ et de 8,80 à $30,90 \%$ pour Nash $(\operatorname{lnQ})$. Les meilleurs critères de Nash obtenus sont ceux calés sur trois ans (1961-1963) avec une période de validation de 26 ans (1964-1990). Le critère de Nash (Q) qui représente l'aptitude du modèle à caler les débits élevés est le plus grand, suivi du Nash $(\sqrt{ } \mathrm{Q})$ et enfin du Nash $(\operatorname{lnQ})$. Ces deux derniers représentent respectivement le calage des débits moyens et faibles. Ainsi, les débits élevés et moyens seront mieux simulés que les débits d'étiage par le modèle GR4J (figure 7).

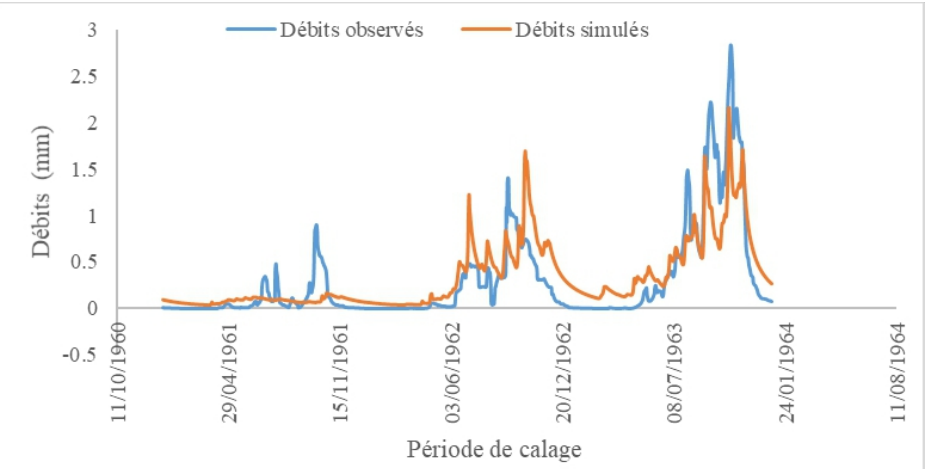

Figure 7 : Calage des débits observés et simulés par le modèle GR4J entre 1961 et 1963 à la station de Bocanda

\subsubsection{Modèle GR2M}

Le tableau III donne les résultats de calage et de validation du modèle GR2M à la station de Bocanda. L'analyse de ce tableau montre que la variation de la période de calage n'a pas d'influence significative sur le paramètre X2. Cependant sur le paramètre $\mathrm{X} 1$, il y a une légère instabilité (figure $8 \mathrm{~A}$ ). 
Comme les résultats du modèle GR4J, les critères de Nash évoluent dans le même sens. Ils diminuent, lorsque la période de calage augmente (figure $8 \mathrm{~B}$ ).

Tableau II : Calage et validation du modèle GR2M sur le sous bassin de Bocanda

\begin{tabular}{lll|ll|lll}
\hline \multirow{2}{*}{$\begin{array}{l}\text { Phase de } \\
\text { calage }\end{array}$} & $\begin{array}{l}\text { Phase de } \\
\text { Validation }\end{array}$ & Paramètres & \multicolumn{3}{l}{ Critères de validation (\%) } \\
& X1 & X2 & Nash & $\begin{array}{l}\text { Nash } \\
\text { (hQ) }\end{array}$ & $\begin{array}{l}\text { Nash } \\
\text { (lnQ) }\end{array}$ \\
\hline $1961-1963$ & $1964-1990$ & 543,11 & 4,18 & 34,30 & 14,30 & $-35,50$ \\
$1961-1964$ & $1965-1990$ & 563,97 & 3,67 & 28,90 & 11,60 & $-32,70$ \\
$1961-1965$ & $1966-1990$ & 557,84 & 3,61 & 27,20 & 10,60 & $-31,40$ \\
$1961-1966$ & $1967-1990$ & 559,35 & 3,62 & 25,20 & 8,60 & $-31,90$ \\
$1961-1967$ & $1968-1990$ & 585,59 & 3,19 & 25,20 & 8,20 & $-32,60$ \\
$1961-1968$ & $1969-1990$ & 592,97 & 3,21 & 36,90 & 12,90 & $-33,70$ \\
$1961-1969$ & $1970-1990$ & 577,56 & 3,18 & 36,00 & 11,50 & $-37,00$ \\
$1961-1970$ & $1971-1990$ & 573,17 & 3,12 & 34,20 & 8,60 & $-41,60$ \\
\hline
\end{tabular}

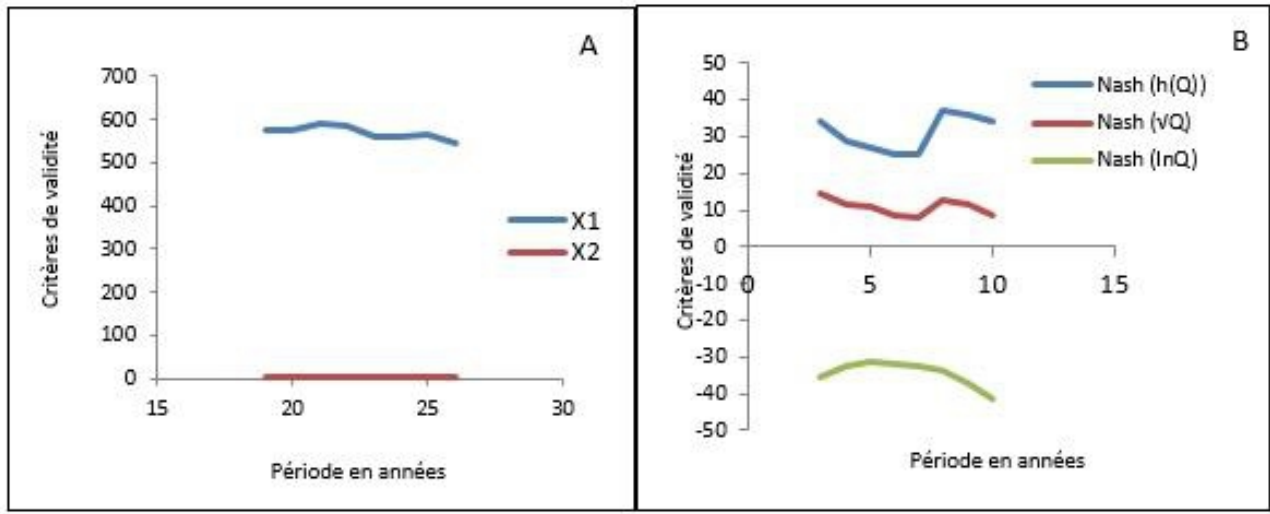

Figure 8 : Evolution des paramètres (A) et des critères de validité (B) du modèle GR2M à la station de Bocanda

Les critères de Nash sont très faibles. Ils évoluent de 25,20 à 36 pour Nash(Q), de 08,20 à 14,30 pour Nash ( $\sqrt{ } \mathrm{Q})$ et de -41 à $-31,40$ pour Nash $(\ln Q)$. Les meilleurs critères de Nash obtenus sont ceux calés sur trois ans (1961-1963) avec une période de validation de 26 ans (1964-1990). La figure 9 présente les débits observés et simulés sur ces 3 ans. L'analyse de la figure montre que les débits observés et simulés présentent un calage insatisfaisant. 


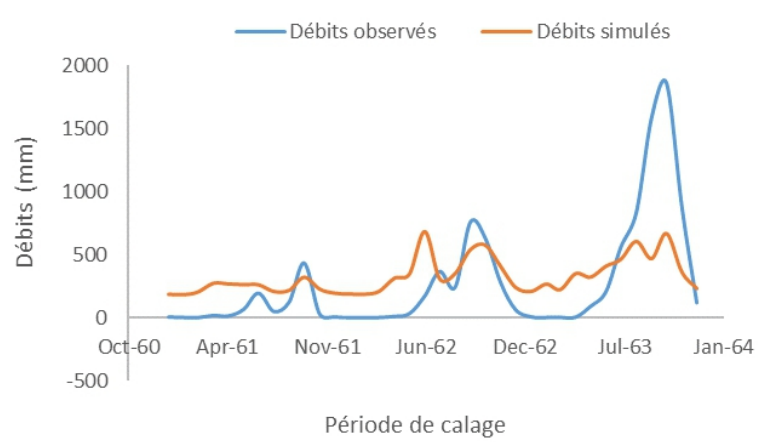

Figure 9 : Calage des débits observés et simulés par le modèle GR2M entre 1961 et 1963 à la station de Bocanda

\subsubsection{Choix du meilleur modèle GR et de la meilleure période}

Les résultats précédents montrent que le modèle GR4J simule mieux les débits de la zone d'étude que le modèle GR2M. Le modèle GR4J simule mieux les débits élevés (période de crue) et les débits moyens. Par contre, il simule mal les débits faibles (période d'étiage). Le modèle GR4J serait mieux indiquer pour faire des prévisions sur les périodes d'inondation. La période de calage doit être relativement courte pour obtenir de meilleurs résultats. Ainsi, la période retenue pour l'étude est de 6 ans de calage, de validation et de vérification (figure 10). Une période de 18 ans permettra donc de déterminer les paramètres optimaux de la zone d'étude.

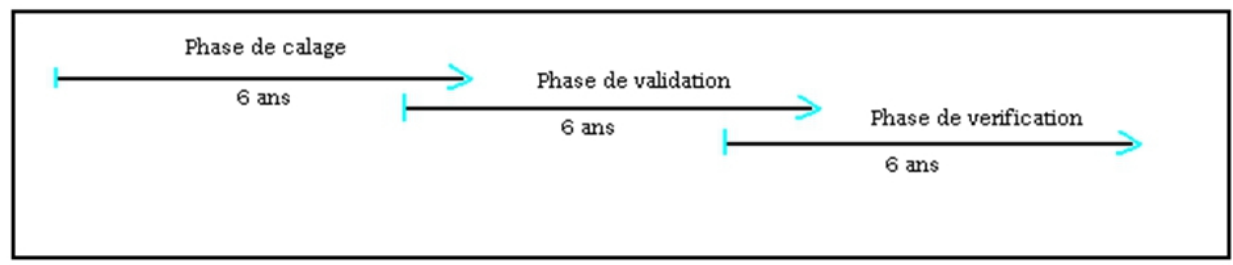

Figure 10 : Période d'application du modèle GR4J

\subsubsection{Application du modèle GR4J dans la zone d'étude}

Les résultats comprennent les phases de calage, de validation et de vérification du modèle GR4J dans les stations de Bocanda et Dimbokro.

\subsubsection{Détermination des paramètres optimaux : phase de calage}

La phase de calage a permis de générer les paramètres optimaux. La période de calage est de 6 ans de 1975 à 1981.

- Station de Bocand

Le tableau IV montre les résultats du calage de 1975 à 1981 à la station de Bocanda. L'analyse du tableau montre que les paramètres générés entre 1977-1978 et entre 1978-1979 donnent les meilleurs critères de validité. 
Tableau IV : Calage du modèle GR4J sur le sous bassin de Bocanda entre 1975-1981

\begin{tabular}{c|cccc|ccc}
\hline \multirow{2}{*}{$\begin{array}{c}\text { Période de } \\
\text { calage }\end{array}$} & \multicolumn{4}{c}{ Paramètres générés } & \multicolumn{3}{c}{ Critères de validité (\%) } \\
& $\mathrm{X} 1$ & $\mathrm{X} 2$ & $\mathrm{X} 3$ & $\mathrm{X} 4$ & $\mathrm{Nash}(\mathrm{Q})$ & $\mathrm{Nash}(\sqrt{ } \mathrm{Q})$ & Nash $(\log \mathrm{Q})$ \\
\hline $1975-1976$ & 261,52 & $-43,52$ & 129,64 & 4,05 & 51,00 & 37,30 & 7,90 \\
$1976-1977$ & 1085,34 & $-2,62$ & 87,17 & 2,19 & 17,50 & 7,60 & $-50,00$ \\
$1977-1978$ & $\mathbf{2 6 1 , 9 7}$ & $\mathbf{- 3 6 , 4 2}$ & $\mathbf{1 3 4 , 2 6}$ & $\mathbf{4 , 2 4}$ & $\mathbf{6 2 , 6 0}$ & $\mathbf{6 3 , 6 0}$ & $\mathbf{6 6 , 9 0}$ \\
$1978-1979$ & $\mathbf{6 9 2 , 9 6}$ & $\mathbf{- 5 , 7 5}$ & $\mathbf{2 6 9 , 0 5}$ & $\mathbf{5 , 2 3}$ & $\mathbf{6 1 , 0 0}$ & $\mathbf{5 5 , 3 0}$ & $\mathbf{2 3 , 3 0}$ \\
$1979-1980$ & 577,86 & 0,23 & 60,79 & 2,31 & 55,70 & 47,70 & 15,50 \\
$1980-1981$ & 1338,16 & $-1,56$ & 41,32 & 2,21 & 18,90 & 27,40 & 15,70 \\
\hline
\end{tabular}

- Station de Dimbokro

Les résultats du calage de 1975 à 1981 à la station de Dimbokro sont consignés dans le tableau V. Les paramètres générés entre 1977-1978 et entre 1979-1980 donnent des critères de validité satisfaisants.

Tableau III : Calage du modèle GR4J sur le sous bassin de Dimbokro entre 1975-1981

\begin{tabular}{c|cccc|ccc}
\hline $\begin{array}{c}\text { Période de } \\
\text { calage }\end{array}$ & \multicolumn{3}{|c|}{ Paramètres générés } & \multicolumn{3}{c}{ Critères de validité (\%) } \\
& $\mathrm{X} 1$ & $\mathrm{X} 2$ & $\mathrm{X} 3$ & $\mathrm{X} 4$ & $\begin{array}{c}\text { Nash } \\
(\mathrm{Q})\end{array}$ & $\begin{array}{c}\text { Nash } \\
(\sqrt{ } \mathrm{Q})\end{array}$ & $\begin{array}{c}\text { Nash } \\
(\log \mathrm{Q})\end{array}$ \\
\hline $1975-1976$ & 164,99 & $-14447,70$ & 4201,64 & 3,84 & 7,70 & 3,00 & $-21,90$ \\
$1976-1977$ & 678,46 & 0,39 & 25,41 & 1,24 & 27,30 & 12,60 & $-46,80$ \\
$1977-1978$ & $\mathbf{7 2 1 , 7 7}$ & $\mathbf{- 1 6 , 1 6}$ & $\mathbf{9 0 , 5 0}$ & $\mathbf{3 , 1 9}$ & $\mathbf{6 0 , 4 0}$ & $\mathbf{6 9 , 0 0}$ & $\mathbf{6 6 , 5 0}$ \\
$1978-1979$ & 454,27 & $-7,67$ & 296,60 & 10,00 & 59,00 & 50,00 & 15,40 \\
$1979-1980$ & $\mathbf{1 4 0 , 8 7}$ & $\mathbf{- 3 , 5 5}$ & $\mathbf{1 4 4 , 2 3}$ & $\mathbf{9 , 6 9}$ & $\mathbf{8 6 , 8 0}$ & $\mathbf{7 3 , 9 0}$ & $\mathbf{3 5 , 4 0}$ \\
$1980-1981$ & 621,55 & $-5,33$ & 114,15 & 3,52 & 53,80 & 58,30 & 41,30 \\
\hline
\end{tabular}

Les phases de validation et de vérification vont soutenir les résultats obtenus lors du calage.

\subsubsection{Détermination des paramètres optimaux : phase de validation et de vérification}

Les résultats des phases de validation et de vérification pour les stations sont présentés à l'aide de tableaux.

- Station de Bocanda

Le tableau VI présente les critères de validité des paramètres optimaux générés lors du calage. L'analyse montre des valeurs de Nash en général satisfaisantes pour la phase de validation. Mais pour la phase de vérification, seuls les paramètres optimaux du calage 1978-1979 donnent des valeurs de Nash satisfaisantes. 
Tableau IVI : Validation et vérification du modèle GR4J sur le sous bassin de Bocanda

\begin{tabular}{ccccccc}
\hline \multirow{2}{*}{$\begin{array}{c}\text { Période } \\
\text { de calage }\end{array}$} & \multicolumn{2}{c}{ Validation (1981-1987) } & \multicolumn{2}{c}{ Vérification (1987-1993) } \\
& $\begin{array}{c}\text { Nash } \\
(\mathrm{Q})\end{array}$ & Nash $(\sqrt{ } \mathrm{Q})$ & Nash $(\log \mathrm{Q})$ & Nash $(\mathrm{Q})$ & Nash $(\sqrt{ } \mathrm{Q})$ & Nash log $(\mathrm{Q})$ \\
\hline $1975-1976$ & $-0,91$ & $-2,45$ & $-2,16$ & 0,06 & 0,20 & 0,30 \\
$1976-1977$ & $\mathbf{0 , 6 7}$ & $\mathbf{0 , 7 7}$ & $\mathbf{0 , 8 0}$ & 0,47 & 0,59 & 0,25 \\
$1977-1978$ & $-1,00$ & $-2,51$ & $-2,33$ & 0,12 & 0,36 & 0,46 \\
$1978-1979$ & $\mathbf{0 , 7}$ & $\mathbf{0 , 8 1}$ & $\mathbf{0 , 6 0}$ & $\mathbf{0 , 7 1}$ & $\mathbf{0 , 7 3}$ & $\mathbf{0 , 6 1}$ \\
$1979-1980$ & $\mathbf{0 , 6 8}$ & $\mathbf{0 , 7 8}$ & $\mathbf{0 , 8 1}$ & 0,52 & 0,46 & $-0,20$ \\
$1980-1981$ & $\mathbf{0 , 6 6}$ & $\mathbf{0 , 7 2}$ & $\mathbf{0 , 7 3}$ & 0,46 & 0,56 & 0,27 \\
\hline
\end{tabular}

La figure 11 montre le calage en phase de validation. Le modèle ne donne pas des critères de Nash très satisfaisants ou excellents parce qu'il sous-estime les débits élevés et il surestime les débits faibles. Ces écarts sont à prendre en compte dans le cas des prévisions par ce modèle.

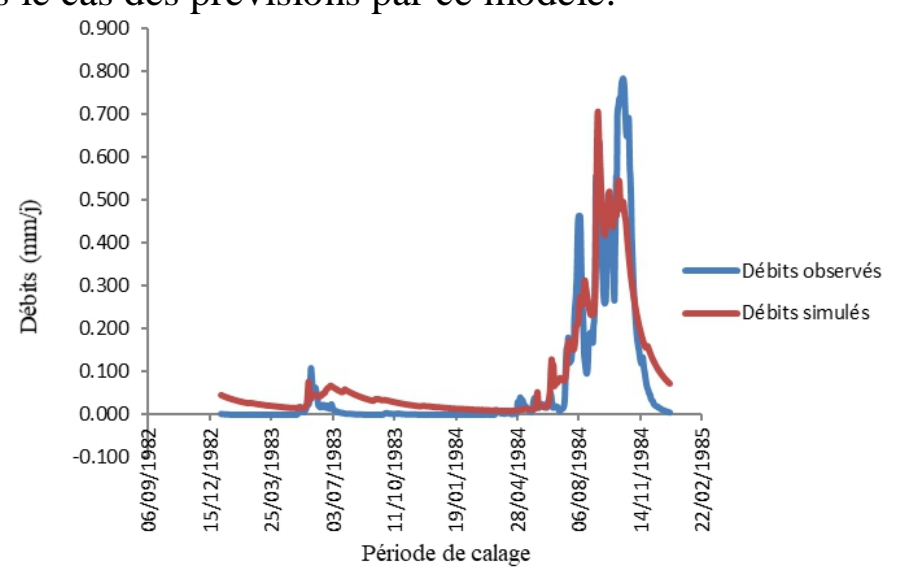

Figure 11 : Phase de validation du GR4J à la station de Bocanda

Les paramètres optimaux retenus sont ceux générés en 1978-1979. Le coefficient d'échange X2 (tableau VII) est négatif. Ce résultat traduit que le N'zi se vide dans le bassin de Bocanda au profit des eaux souterraines.

Tableau VI : Paramètres optimaux retenus pour la station de Bocanda avec le modèle GR4J

\begin{tabular}{|c|c|c|c|}
\hline \multicolumn{2}{|c|}{ Paramètres optimaux } & \multicolumn{2}{|c|}{ Critères de validité } \\
\hline X1 & 692,96 & $\operatorname{Nash}(\mathbf{Q})$ & 0,7 \\
\hline $\mathbf{X} 2$ & $-5,75$ & $\operatorname{Nash}(\sqrt{ } Q)$ & 0,81 \\
\hline $\mathrm{X3}$ & 269,05 & Nash $\log (Q)$ & 0,6 \\
\hline $\mathrm{X} 4$ & 5,23 & & \\
\hline
\end{tabular}

- Station de Dimbokro 
Les résultats de la phase de validation et de vérification sont consignés dans le tableau VIII. L'analyse du tableau montre des valeurs de Nash inférieures à $60 \%$. Ce résultat signifie que les paramètres optimaux générés ne reflètent pas la réalité de ce bassin. Le modèle GR4J ne donne pas des résultats satisfaisant pour le bassin de Dimbokro.

Tableau VIIVI : Validation et vérification du modèle GR4J sur le sous bassin de Dimbokro

\begin{tabular}{ccccccc}
$\begin{array}{c}\text { Période de } \\
\text { calage }\end{array}$ & \multicolumn{3}{c}{ Validation (1981-1987) } & \multicolumn{3}{c}{ Vérification (1987-1993) } \\
& $\begin{array}{c}\text { Nash } \\
(\mathrm{Q})\end{array}$ & $\begin{array}{c}\text { Nash } \\
(\sqrt{ } \mathrm{Q})\end{array}$ & $\begin{array}{c}\text { Nash } \\
(\log \mathrm{Q})\end{array}$ & $\begin{array}{c}\text { Nash } \\
(\mathrm{Q})\end{array}$ & $\begin{array}{c}\text { Nash } \\
(\sqrt{ } \mathrm{Q})\end{array}$ & $\begin{array}{c}\text { Nash } \\
(\log \mathrm{Q})\end{array}$ \\
\hline $1975-1976$ & -128 & $-2,32$ & $-2,51$ & & & \\
$1976-1977$ & $-0,81$ & 0,51 & 0,25 & & & \\
$1977-1978$ & $\mathbf{0 , 3 2}$ & $\mathbf{0 , 4 4}$ & $\mathbf{0 , 5 8}$ & 0,25 & 0,41 & 0,5 \\
$1978-1979$ & $-0,77$ & $-0,55$ & $-0,87$ & & & \\
$1979-1980$ & $-0,19$ & 0,35 & 0,05 & & & \\
$1980-1981$ & $-0,48$ & 0,25 & 0,09 & & & \\
\hline
\end{tabular}

Le modèle GR4J simule de façon satisfaisante les écoulements du bassin de Bocanda contrairement à ceux du bassin de Dimbokro.

\subsection{Discussion}

Les résultats de la simulation hydrologique ont montré que le modèle GR4J donne des résultats plus satisfaisants que le modèle GR2M. Le choix du modèle pluie-débit repose tout d'abord sur la performance du modèle (Mouelhi, 2003). Mais ces résultats ont permis de comprendre que la performance d'un modèle ne réside pas en sa complexité. En effet, le modèle GR4J est un modèle à paramètres restreints par rapport aux modèles globaux qui sont complexe à cause des nombreux paramètres qu'ils nécessitent. L'utilisation des modèles globaux nécessite une parfaite maîtrise des états de surface et surtout une mesure in situ des pluies par l'utilisateur. Or les données utilisées dans cette étude ne mentionnent pas la durée des pluies. Par contre, le modèle GR4J est un modèle régional qui calcule les débits à l'exutoire au pas de temps journalier. Il est facile à utiliser et garantit de bons résultats. Le choix du modèle réside donc sur les objectifs de l'étude. Ainsi, Mouelhi (2003) suggère l'utilisation du modèle GR4J pour générer les débits plutôt que d'utiliser le modèle GR2M avec des données mensuelles ; les résultats de cette étude sont en accord avec ceux de Kouassi (2007) qui trouve des critères de validité faibles (en dessous de $60 \%$ ) pour la station de Bocanda avec le modèle GR2M. Au sud-est du pays, dans la région d'Aboisso, Dibi (2008) trouve des critères de validité faibles (Nash calage $=72,40 \%$; Nash validation $=52,40 \%$ ) sur la Bia avec le modèle GR2M. Adja et al. (2019) trouvent également des critères de validité très faibles (voire négatif) dans le sous-bassin Versant du 
Kouto (Nord de la Côte d'Ivoire) avec le modèle GR2M. De même Sambou et al. (2011) trouvent de meilleurs résultats avec le modèle GR4J que le modèle GR2M. Cependant, Traoré et al. (2014) dans la station de Medina gounass au Sénégal évaluent des critères de validité satisfaisants pour les deux modèles. Ils trouvent des valeurs comprises entre 80 et $90 \%$ pour le modèle GR2M et le modèle GR4J en phase de validation et de vérification. Bodian et al. (2012) également sur le haut bassin du fleuve Sénégal trouvent des critères de validité satisfaisants pour le modèle GR2M. Ces critères sont en général supérieurs à $60 \%$ (validation et de vérification) sur les trois stations étudiées. En dehors du paramètre X1, la variation de la période de calage n'a pas d'influence sur les paramètres optimaux. Sambou et al. (2011), constatent des résultats similaires dans le sous bassin du Bafing au Sénégal. L'incapacité du modèle GR4J à simuler les débits d'étiage a également été observé par Dezetter et $a l$. (2010) sur le bassin du Niger à Koulikoro et par Bodian et al. (2012) au Sénégal avec le modèle GR2M. Par ailleurs, Ouermi et al. (2015) et Bourgin (2019) montrent que les performances du modèle GR2M en Afrique de l'ouest sont meilleures si on cale le modèle en période sèche et qu'on l'applique en période humide que l'inverse. En dehors des états de surface qui n'ont pas été pris en compte, les résultats insatisfaisants obtenus sur le bassin de Dimbokro seraient dû à sa grande variabilité pluviométrique contrairement au bassin de Bocanda qui présente une série pluviométrique presque homogène.

Le coefficient d'échange négatif $(-5,75)$ pourrait expliquer la raison pour laquelle le N'zi se vide dans le bassin de Bocanda, alors que la baisse de la pluviométrie est négligeable (Fossou et al. (2014).

\section{Conclusion}

L'étude de la modélisation hydrologique avec les modèles hydrologiques GR2M et GR4J a montré que le modèle GR4J simule mieux les écoulements de la zone d'étude que le modèle GR2M. La période de calage doit être relativement courte pour obtenir de meilleurs résultats. En effet, les critères de Nash obtenus pour le modèle GR4J varient de 40,80 à 67,40\% pour les débits élevés, de 34,10 à $61 \%$ pour les débits moyens et de 8,80 à $30,90 \%$ pour les débits faibles. Cependant, les critères de Nash du modèle GR2M sont très faibles. Ils évoluent de 25,20 à $36,90 \%$ pour les débits élevés, de 08,20 à $14,30 \%$ pour les débits moyens et de $-41,60$ à $-31,40 \%$ pour les débits faibles. En fait, les modèles simulent de façon acceptable la variation des débits observés en termes d'allure, mais ce sont les écarts qui existent entre les débits observés et simulés qui les distinguent. Ainsi, le choix d'un de ces modèles dépendra des objectifs fixés, d'une analyse et d'une interprétation basées sur les travaux ci-dessus et antérieurs. Le coefficient d'échange X2 est négatif. Ce résultat pourrait expliquer la raison pour laquelle le N'zi se vide dans le bassin 
de Bocanda, alors que la baisse de la pluviométrie est négligeable. Le modèle GR4J serait mieux indiquer pour faire des prévisions sur les périodes d'inondation à partir des modèles climatiques du GIEC (Groupe d'experts intergouvernemental sur l'évolution du climat). Ce modèle pourrait être aussi utilisé dans de nombreuses applications d'ingénierie et de gestion de la ressource en eau, telles que le dimensionnement et la gestion d'ouvrages, la détection d'impact, etc.

\section{References:}

1. Adja, M. G., Djémin, E. J., N’Dri, O. F.A., Tohouri, P., Kouamé, K. J., Jourda, J. P., 2019. “Apport de l'Analyse Hydrologique et de la Modélisation de la Relation Pluie-Débit dans le Suivi Quantitatif des Ressources en Eau dans un Contexte de Variabilité Climatique : Cas du Sous-Bassin Versant du Kouto (Nord de la Côte d'Ivoire) ", European Journal of Scientific Research, Vol 154 N² 2, pp. 192-212.

2. Andreassian, V., Parent, E., and Michel, C., 2003. "A distribution-free test to detect gradual changes in watershed behavior", Water resources research, Vol $39 \mathrm{~N}^{\circ}$ 9, pp. 11.

3. Ardoin, B. S., 2004. "Variabilité hydroclimatique et impacts sur les ressources en eau de grands bassins hydrographiques en zone soudanosahélienne", Thèse de Doctorat, Université de Montpellier II, France, $437 \mathrm{p}$.

4. Ardoin, B. S., Lubes, N. H., Servat, E., Dezetter, A., et Boyer, J. F., 2003. "Analyse de la persistance de la sécheresse en Afrique de l'Ouest : caractérisation de la situation de la décennie 1990”, IAHS Publication, $\mathrm{N}^{\mathrm{o}} 278$, pp. 223-228.

5. Bodian, A., Dezetter, A., et Dacosta, H., 2012. "Apport de la modélisation pluie-débit pour la connaissance de la ressource en eau : application au haut bassin du fleuve Sénégal", Climatologie, vol. 9, pp 109-125.

6. Bourgin P,Y., 2019. "Modèle intégré du fonctionnement hydrologique du bassin versant du Sassandra" La Houille Blanche, 5-6, pp 124-139.

7. Dezetter, A., Paturel, J-E., Ruelland, D., Ardoin, B. S., Ferry, L., Mahe, G., Dieulin, C., Servat, E., 2010. "Prise en compte des variabilités spatio-temporelles de la pluie et de l'occupation du sol dans la modélisation semi-spatialisée des ressources en eau du haut fleuve Niger. In 'Global Change : facing risks and threats to water resources”, proceedings of the sixth world FRIEND conference, Wallingford, IAHS, pp. 544-552.

8. Dibi, B., 2008. "Cartographie des sites potentiels d'implantation des points d'eau dans le département d'Aboisso (Sud-Est de la Côte 
d'Ivoire) : apport du SIG et de l'analyse multicritère", Thèse de Doctorat de l'Université de Cocody, Côte d'Ivoire, $164 \mathrm{p}$.

9. Fossou, R. M. N., Soro, N., Traore, V. B., Lasm, T., Sambou, S., Soro, T., Orou, R. K., Cisse, M.T., Kane, A., 2014. "Variabilité climatique et son incidence sur les ressources en eaux de surface : cas des stations de Bocanda et de Dimbokro, Centre-Est de la Côte d'Ivoire en Afrique de l'Ouest", Afrique SCIENCE, Vol $10 \mathrm{~N}^{\circ}$ 4, pp. 118 -134.

10. Fourmigue, P., et Lavabre, J., 2005. "Prévision de crues avec le modèle conceptuel pluie-débit GR3H”, Revue des Sciences de l'Eau, Vol 18 $\mathrm{N}^{\mathrm{o}} 1$, pp. 87-102.

11. Goula, B.T.A., Savané, I., Konan, B., Fadika, V. et Kouadio, G.B., 2005. "Étude comparative de l'impact de la variabilité climatique sur les ressources en eau des bassins du N'Zo et du N'Zi en Côte d'Ivoire", Poster AOC, Séminaire International, 23-24 novembre 2005, pp. 5-36.

12. Koffi, Y.B., Lasm, T., Ayral, P.A., Anne, J., Kouassi, A.M., Assidjo, E., et Biemi, J., 2007. "Optimization of multi-layers perceptron's models with algorithms of first and second order. Application to the modelling of rainfall-rainoff relation in Bandama Blanc catchment (north of Ivory Coast)", European Journal of Scientific Research, Vol $17 \mathrm{n}^{\circ} 3$, pp. 13-28.

13. Kouassi, A. M., 2007. "Caractérisation d'une modification éventuelle de la relation pluie débit et ses impacts sur les ressources en eau en Afrique de l'Ouest : cas du bassin versant du N'zi (Bandama) en Côte d'Ivoire", Thèse de Doctorat, Université de Cocody-Abidjan, Côte d'Ivoire, $210 \mathrm{p}$.

14. Leblond, P., 1984. "Contribution aux études hydrogéologiques en Côte d'Ivoire. Région de Yamoussoukro (Station expérimentale de l'ENSTP)", Thèse de 3è cycle, Université de Bordeaux 1, France, 150 p.

15. Makhlouf, Z., 1994. "Compléments sur le modèle pluie-débit GR4J et essai d'estimation de ses paramètres", Thèse de Doctorat, Université Paris XI Orsay, France 426 p.

16. Michel, C., 1983. "Que peut-on faire en hydrologie avec un modèle conceptuel à un seul paramètre ?" La Houille Blanche, $\mathrm{n}^{\circ} 1$, pp. 3944.

17. Mouelhi, C., (2003). "Vers une chaîne cohérente de modèles pluiedébit conceptuels globaux aux pas de temps pluriannuel, annuel, mensuel et journalier". Thèse de Doctorat, Ecole nationale du génie rural des eaux et forêts de Paris, France, 274p.

18. Ouedraogo, M., 2001. "Contribution à l'étude de l'impact de la variabilité climatique sur les ressources en eau en Afrique de l'Ouest. Analyse des conséquences d'une sécheresse persistante : normes 
hydrologiques et modélisation régionale". Thèse de Doctorat, Université de Montpellier II, France, 257p.

19. Ouedraogo, M., Servat, E., Paturel, J.E., Lubes, N. H et Masson, J M., 1998. "Caractérisation d'une modification éventuelle de la relation pluie-débit autour des années 1970 en Afrique de l'Ouest et centrale non sahélienne", IAHS Pub. 252 pp. 315-321.

20. Perrin, C. (2000). "Vers une amélioration d'un modèle global pluiedébit au travers d'une approche comparative", Thèse de Doctorat, INPG (Grenoble)/Cemagref (Antony), $530 \mathrm{p}$.

21. Perrin, C., Michel, C., et Andreassian, V., 2007. "Modèles hydrologiques du Génie Rural (GR) ”, Cemagref, UR Hydrosystèmes et Bioprocédés Parc de Tourvoie, 16p.

22. Riad, S., 2003. "Typologie et analyse hydrologique des eaux superficielles à partir de quelques bassins versants représentatifs du Maroc", Thèse de Doctorat, Université des Sciences et Technologies de Lille (France) et Université Ibnou Zohr d'Agadir (Maroc), 154 p.

23. Rojas, S. C., 2005. "Quelles connaissances hydrométriques minimales pour définir les paramètres d'un modèle pluie-débit ?", Thèse de Doctorat, Cemagref (Antony), ENGREF (Paris), France, 319 pp.

24. Sambou, S., Boye, M., Malang, B. A., Malada, N. E. N., Bodian. A., Mussa, K., Adma, M et Bamba, F., 2011. "Calage et validation des modèles hydrologiques GR2M et GR4J sur le bassin du Bafing en amont de Bafing-Mankana : vers l'étude de l'impact du climat sur les ressources en eau de la retenue de Manantali”, $6{ }^{\text {ème édition des journées }}$ scientifiques du $2 \mathrm{iE}$.

25. Savane, I., Coulibaly, K. M., Gioan, P. (2001). "Variabilité climatique et ressources en eaux souterraines dans la région semi-montagneuse de Man”, Sécheresse, Vol 12 n4, pp. 231-237.

26. Traore, V. B., Sambou, S., Tamba, S., Fall, S., Diaw, A. T. et Cisse, M. T., 2014. "Calibrating the rainfall-runoff model GR4J and GR2M on the Koulountou river basin, a tributary of the Gambia river", American Journal of Environmental Protection Vol 3 n 1 pp. 36-44.

27. Yao, D., Delor, C., Gadou, G., Kohou, P., Okou, A., Konate, S., et Diaby, I., 1990 "Notice explicative de la carte géologique feuille de M'Bahiakro".

28. Yao, D., Delor, C., Gadou, G., Kohou, P., Okou, A., Konate, S. et Diaby, I., 1995 "Notice explicative de la carte géologique feuille de Dimbokro." 